\title{
Ageing and Changes in Protein Conformation in the Human Lens: A Raman Microspectroscopic Study
}

\author{
ITTE SIEBINGA ${ }^{a, b}$, GIJS F. J. M. VRENSENª, KEES OTTO ${ }^{b}$, GERWIN J. PUPPELS \\ FRITS F. M. DE MUL ${ }^{b}$ AND JAN GREVE \\ ${ }^{a}$ Department of Morphology, The Netherlands Ophthalmic Research Institute, P.O. Box 12141, \\ 1100 AC Amsterdam, and 'Department of Applied Physics, University of Twente, P.O. Box 217. \\ 7500 AE Enschede, The Netherlands
}

(Received Norwich 3 April 1991 and accepted in revised form 19 June 1991)

\begin{abstract}
Using confocal Raman microspectroscopy with laser light of $660 \mathrm{~nm}$ the secondary and tertiary conformation of crystallins was studied in human lenses of varying age (20-75 years). Differences in cortical and nuclear proteins in individual lenses and among lenses of different age and differences between small equatorial opacities and adjacent clear sites were analysed using a difference spectrum approach. Intensity calibration allows assessment of local variations in protein content.

The main findings and conclusions are as follows. (1) Irrespective of the age of the lens nuclear proteins proved to contain more aromatic amino acids, i.e. tryptophan. tyrosine and phenylalanine. This change most probably reflects differences in crystallin composition between nucleus and cortex as described in biochemical studies. (2) Changes in the amide bands indicate a more pronounced $\beta$-sheet conformation of the nuclear proteins. Taking into account available biochemical evidence this most probably reflects a true post-translational change in the secondary conformation of the crystallins. (3) Cortical proteins in 'old' and 'young' lenses are largely identical indicating that ageing is not accompanied by gross alterations in the transcription/translation of the crystallin genes. (4) 'Old' and 'young' nuclear proteins deviate with respect to the amount of aromatic amino acids, being more abundant in 'young' nuclear proteins. (5) Proteins in small early opacities do not exhibit alterations in conformation. (6) A pronounced peak in the difference spectra in the region $1425-1435 \mathrm{~cm}^{-1}$ for nuclear proteins especially when compared with equatorial cortical proteins may be considered as evidence for the advanced photooxidation of tryptophan and/or deamidation of asparagine from superficial to deep regions of the lens.
\end{abstract}

Key words: Raman spectroscopy; human lens; protein conformation; ageing.

\section{Introduction}

As emphasized by Harding (1981) 'Many manifestations of cataract are exaggerations of changes found in the aging lens'. As, for example, suggested by biochemical and Raman studies the rise in disulphide bridges and concomitant decrease in sulphydryl groups are age-related in many species, leading to the formation of high-molecular-weight proteins and possibly preceding nuclear cataract formation (Harding, 1981; Hoenders and Bloemendal, 1981, 1983; Itoh et al., 1983; Ozaki et al., 1983; Spector, 1985; Yu et al., 1985; Ozaki et al., 1987; Nie et al., 1990). However, human and guinea-pig lenses seem to be an exception as indicated by the Raman study of Yu et al. (1987). Among many other protein changes conformational changes are considered as age-related alterations of lens proteins which may be relevant signs of cataract formation. Since the lens is a lifelong growing organ with no shedding of cellular elements it comprises 'old', i.e. post-translationally altered proteins in the nucleus and 'young', i.e. newly synthesized proteins in the superficial cortex. In addition, the newly synthesized proteins in the

\footnotetext{
* For correspondence and reprint requests.
}

superficial cortex in 'young' and 'old' lenses are synthesized from crystallin genes of different ages and may show age-related transcriptional/translational differences in these genes. Therefore, the study of crystallin changes related to age uses a method allowing locally defined measurements.

For the analysis of protein conformation changes Xray diffraction (XRD), circular dichroism (CD), optical rotary dispersion (ORD), fluorescence spectroscopy (FS) and Raman spectroscopy are powerful tools. A disadvantage of XRD, CD and ORD is that they have to be carried out on isolated proteins in aqueous solution (CD, ORD) or crystal state (XRD). Isolation largely frustrates local discrimination. FS can be carried out in intact lenses (Yu. Bando and Kuck, 1985; Barron. Yu and Kuck, 1987). The information obtained for eye lens proteins is limited to the environment of tyrosine and tryptophan side chains and the method does not seem very well suited for determining the peptide backbone structure (Tu, 1982). Raman spectroscopy provides information on the peptide backbone and its conformation, the presence of disulphide bonds and the sidechains of some amino acids. Since it can be carried out with crystals, powders, aqueous solutions and with intact tissue and has an excellent spatial resolution especially when used in a confocal micro- 
spectroscopic set-up, it seems the most powerful of these tools for the study of age-related changes in lens proteins.

The present study summarizes our Raman investigations on slices of clear human lenses of varying age. The Raman analysis was restricted to the 650$1750 \mathrm{~cm}^{-1}$ spectral region. This region comprises the most relevant information related to the peptide backbone conformation and to the aromatic and nonaromatic sidechains. It does not include the Raman signals of disulphide bonds and sulphydryl groups. However, with respect to age-related changes in these bonds and groups numerous papers have already appeared (Itoh et al., 1983; Ozaki et al., 1983, 1987; Parker, 1983; Yu et al., 1985, 1987; Yu, Bando and Kuck, 1990; Nie et al., 1990).

\section{Materials and Methods}

Raman spectra were recorded with the Confocal Raman Microspectrometer (CRM) developed in the Department of Applied Physics, University of Twente. The Netherlands. A detailed description of the CRM has been given by Puppels et al. (1990, 1991). For lens measurements it was equipped with a water immersion objective (Zeiss, $63 \times$, NA 1.2). Spatial resolution is $0.45 \times 0.45 \times 1.3 \mu \mathrm{m}^{3}$. Laser light of $660 \mathrm{~nm}$ from a DCM-operated dye-laser (Spectra Physics, model $375 \mathrm{~B}$ ), was used for excitation. The dye-laser was pumped by an Argon-ion laser (Coherent Radiation Innova 90). A liquid nitrogen cooled CCDcamera (Wright Instruments Ltd) was used for signal detection. Wave number calibrations were made by recording an indene spectrum at the same instrumental conditions as used for lens measurements. All spectra have been corrected for the wave numberdependent signal detection efficiency of the CRM. The laser power at the sample varied between 5 and $10 \mathrm{~mW}$. However, during one experiment the laser power remained constant.

Human lenses varying in age between 20 and 75 years were obtained from eyes donated for cornea transplantation. For the present study lenses were selected with no or only a few equatorial opacities and minimal discoloration (light yellow). The lenses were extracted within $24 \mathrm{hr}$ post-mortem and were fixed in a solution of $0.08 \mathrm{M}$ cacodylate buffered $1 \%$ paraformaldehyde (pH $7 \cdot 3.310 \mathrm{mosmol})$. As shown in previous studies the post-mortem time and the fixation procedure do not effect the water/protein content of the lenses and have no effect on the tryptophan and tyrosine Raman signals (Bot et al., 1989; Schyns et al., 1990). Lens slices of about $1 \mathrm{~mm}$ thickness were cut along the visual or equatorial axis as described earlier (Bot et al., 1989). They were put on glass slides and covered with coverglasses of $0.17 \mathrm{~mm}$ thickness. A drop of a phosphate-buffered saline solution was used to prevent them from drying.

In seven human lenses Raman spectra were obtained in the $650-1750 \mathrm{~cm}^{-1}$ spectral region in both the nucleus and the anterior cortex. All spectra in the anterior cortex were recorded within $200 \mu \mathrm{m}$ from the lens surface. In order to compare the spectra obtained in the nucleus with the spectra obtained in the anterior cortex difference spectra were calculated. For that the spectra were subtracted after scaling the spectra on the basis of the intensity of the $1450 \mathrm{~cm}^{-1}$ protein band (cf. Otto. de Mul and Greve, 1987). The intensity of the $1450 \mathrm{~cm}^{-1}$ band depends only on the number of protein $\mathrm{CH}_{2}$ and $\mathrm{CH}_{3}$ groups and is insensitive to protein secondary structure (Fasman et al., 1978: Tu, 1982: Ozaki et al., 1983). Since phospholipids account for an extremely small fraction of total lens mass $(<0.2 \%)$ the sensitivity to microenvironmental changes of the long $\mathrm{CH}_{2}$ chains of the fatty acids in the phospholipids will not affect the intensity of the $1450 \mathrm{~cm}^{-1}$ band. In addition, the changes in amino acid composition with age and among crystallins are marginal and will not alter the number of $\mathrm{CH}_{2}$ and $\mathrm{CH}_{3}$ groups (Coghlan and Augusteyn, 1977). It is therefore justified to use this line as an internal standard for protein concentration. The integrated intensity in the interval from 1378 to $1510 \mathrm{~cm}^{-1}$ was calculated and used to calibrate the intensity in the eye lens spectra.

In Fig. 1 an example is shown of two measured spectra obtained in the nucleus and the anterior cortex of a human lens respectively together with the calculated difference spectrum. The spectrum intensity of the protein line used for scaling is indicated by the hatched region in the figure. All difference spectra were calculated in exactly the same way. In order to investigate age-related transcriptional/translational differences within the nucleus and within the cortex. difference spectra were also calculated between the spectra obtained in nuclear and cortical regions of three lenses of varying age. In addition, two human lenses with small opaque spots in their equatorial region were investigated. Raman spectra were measured in and outside the opaque spots and difference spectra were calculated as described above.

In order to compare the depolarization ratios of the Raman bands recorded in and outside opaque spots, spectra were recorded in these regions for two polarization directions. With a polarization filter (Spindler \& Hoyer type $10 \mathrm{~K}$ ) installed in the set-up, spectra were measured with polarization parallel and orthogonal to the polarization of the laser light. By the correction procedure for the wave number detection efficiency of the apparatus the polarization-dependent efficiency was also taken into account.

\section{Results}

Figure 1 gives an example of Raman spectra taken at a nuclear and superficially cortical position along the optical axis in the lens of a 59-year-old donor. In addition, the difference spectrum (nucleus minus 


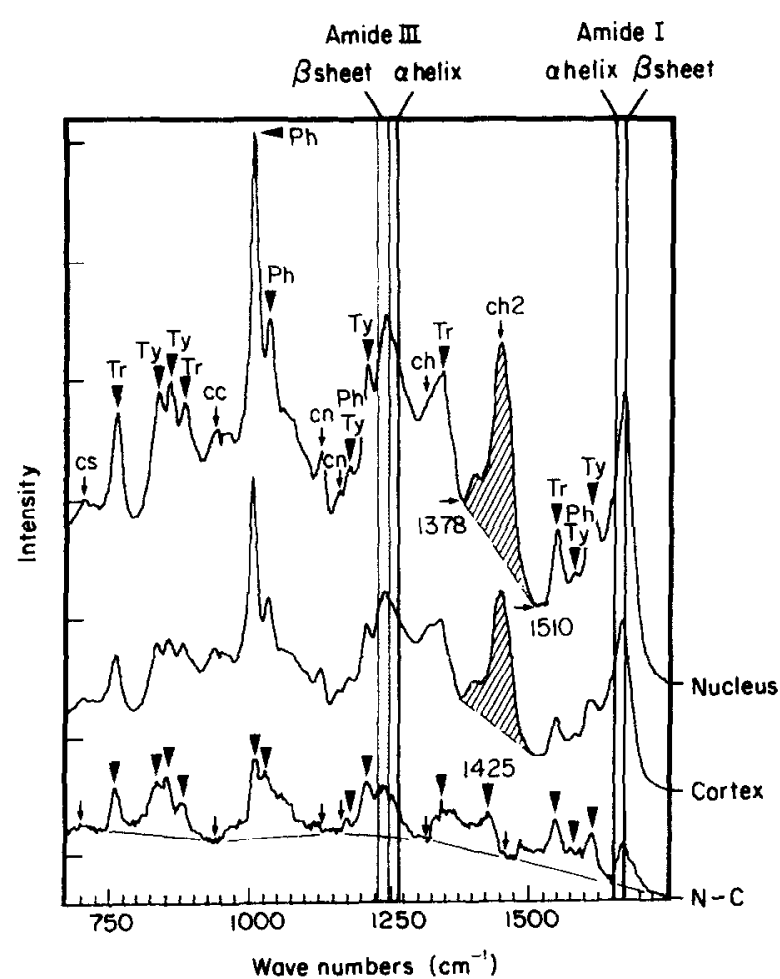

FIG. 1. Raman spectra of a 59-year-old donor lens in the nucleus and anterior superficial cortex. The difference spectrum (A-B) (nucleus minus cortex) was obtained as indicated in Materials and Methods. The difference spectrum was obtained after scaling on the $1450 \mathrm{~cm}^{-1}$ band. Tr. Tryptophan; Ty, tyrosine; $\mathrm{Ph}$, phenylalanine; cs, CSvibration; cc, CC-vibration; cn, $\mathrm{CN}$-vibration; ch, $\mathrm{CH}$ vibration: $\mathrm{ch} 2, \mathrm{CH}_{2}$-vibration.

cortex) is given, obtained as described under Materials and Methods. Figure 2 illustrates the intraindividual difference spectra of all lenses analysed in this study. The Raman vibrations of the protein backbone and non-aromatic sidechains (cs, $725 \mathrm{~cm}^{-1} ; \mathrm{cc}, 936 \mathrm{~cm}^{-1}$; cn, 1129 and $1159 \mathrm{~cm}^{-1}$; ch, $1322 \mathrm{~cm}^{-1}$ ) did not show peaks in the difference spectra indicating that the backbone and non-aromatic sidechains were not significantly different between cortex and nucleus. The Raman signals of the aromatic amino acids tyrosine (Ty), tryptophan ( $\mathrm{Tr}$ ) and phenylalanine $(\mathrm{Ph})$ proved to be in excess in the nucleus at all assigned wave numbers. The amide vibrations at $1227-1264 \mathrm{~cm}^{-1}$ (Amide III) and at $1660-1673 \mathrm{~cm}^{-1}$ (Amide I) exhibited consistent changes in all lenses studied. The amide vibrations can be disjoined in peaks reflecting the $\alpha$-helix conformation at $1660 \pm 4 \mathrm{~cm}^{-1}$ (Amide I) and at $1265 \mathrm{~cm}^{-1}$ (Amide III) and peaks reflecting the $\beta$-sheet conformation at $1673 \pm 2 \mathrm{~cm}^{-1}$ (Amide I) and at $1227-1240 \mathrm{~cm}^{-1}$ (Amide III) (see Parker, 1983 and references therein). It proved (Figs. 1 and 2) that the lens nuclcus consistently has an increased Raman signal for the amide vibrations of the $\beta$-sheet. Finally, all spectra exhibited a peak with a maximum at $1425 \mathrm{~cm}^{-1}$.

Figure 3 illustrates the interindividual difference spectra among lenses of different age at two optical axis positions (nuclear and superficial cortical). The

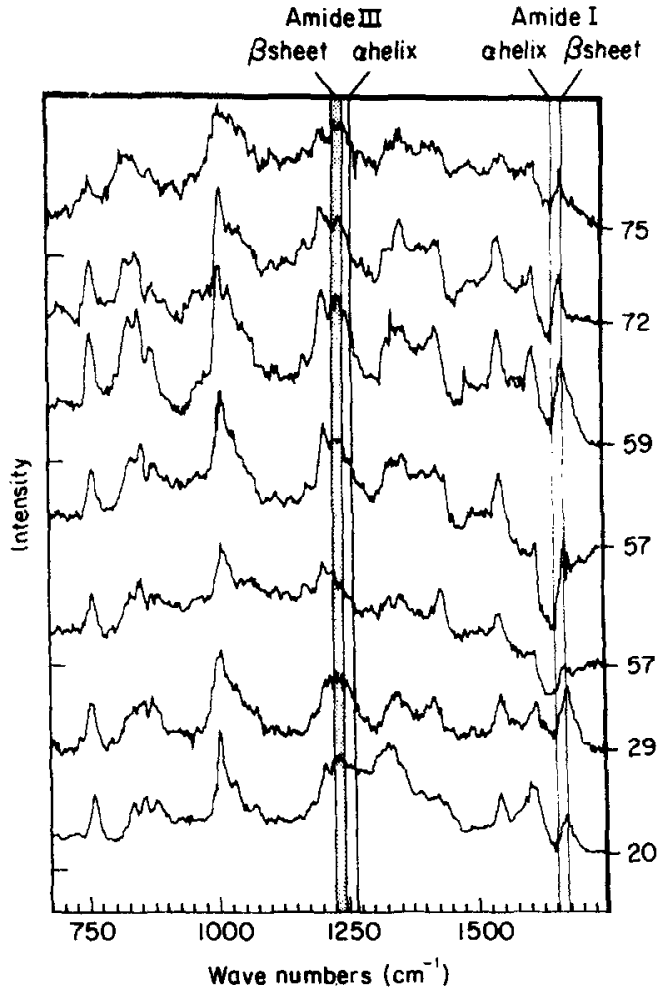

FIG. 2. Difference Raman spectra (nucleus minus cortex) of the lenses analysed in this study. The age in years of the lenses is indicated on the right-hand $y$-axis.

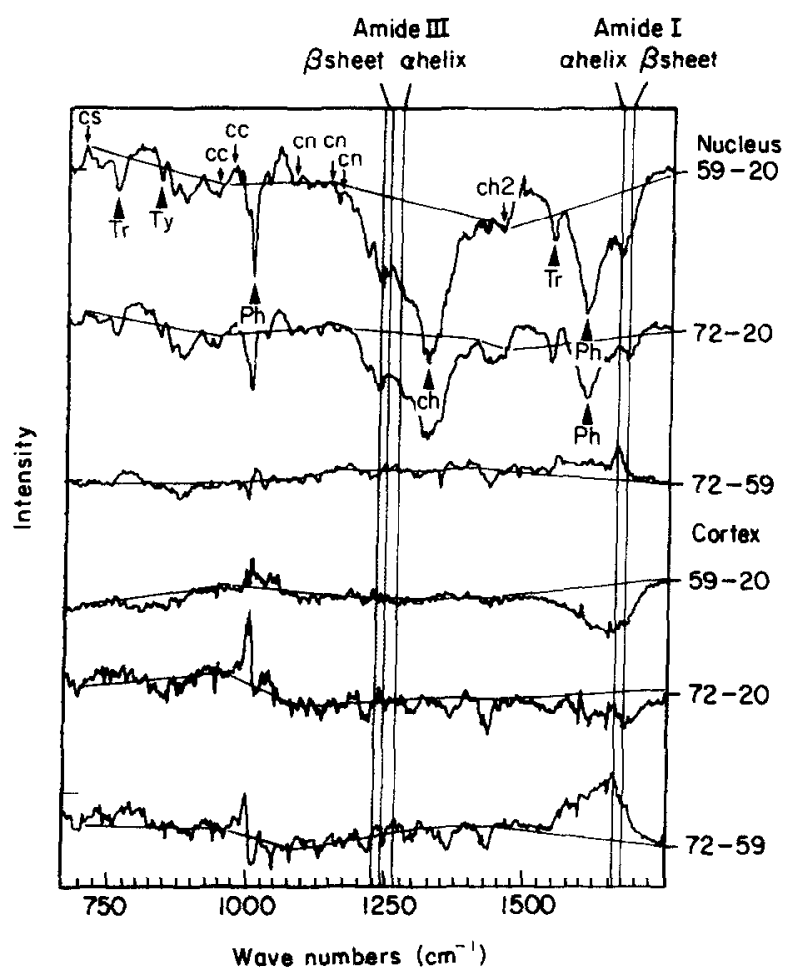

FIG. 3. Difference spectra between three lenses aged 20, 59 and 72 years at a nuclear location and an anterior superficial cortical position.

comparison was deliberately restricted to three lenses analysed on the same day, i.e. under exactly identical instrumental conditions, especially regarding the laser power. The difference spectra at the superficial cortical 

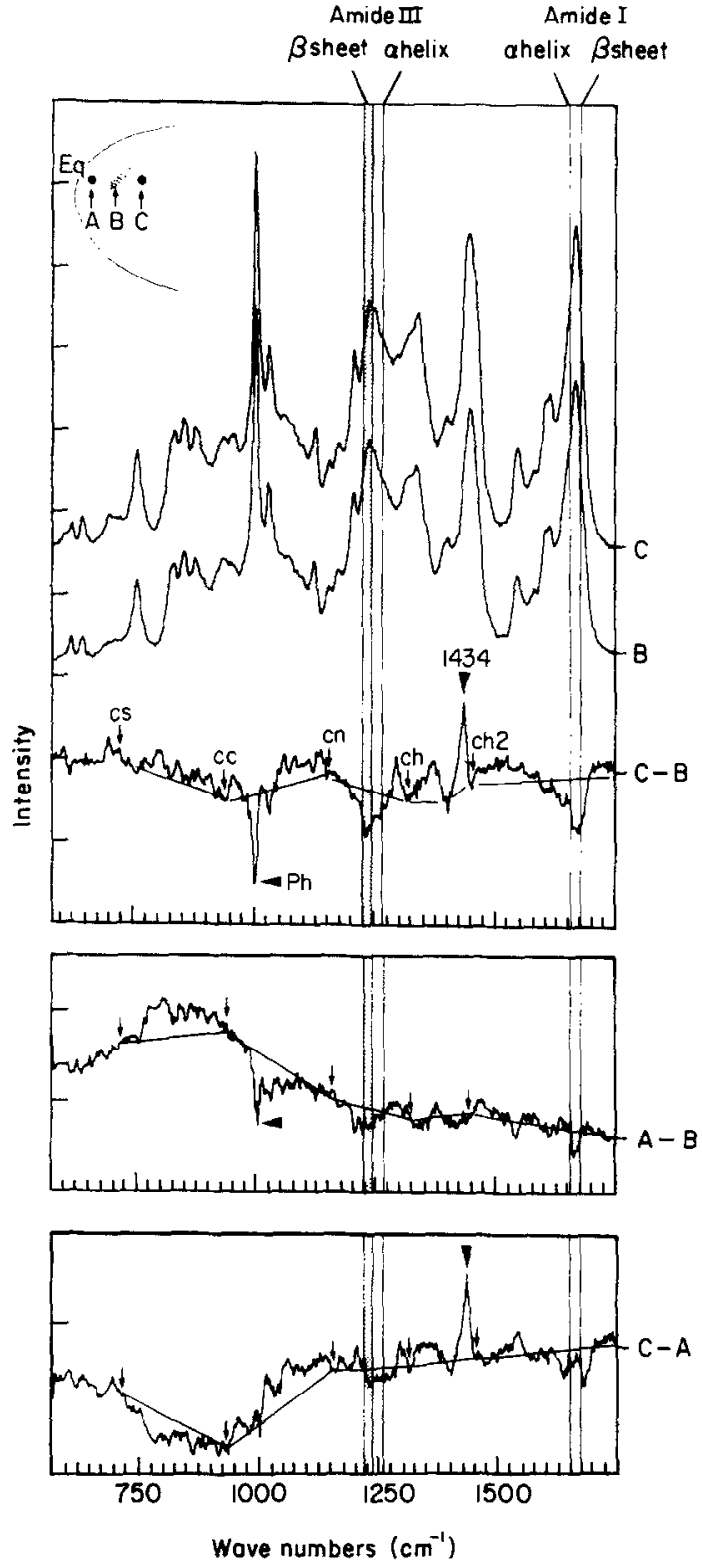

Frg. 4. Panel illustrating the Raman analysis of an opaque spot (B) and two closely adjacent transparent sites (A, C). Eq, Lens equator.

locations did not show consistent differences. The nuclear locations, however, showed clear-cut differences between the three difference spectra. Comparison between 59-year-old and 72-year-old lenses (72-59) revealed that the Raman vibrations were nearly identical, with no obvious peaks. Comparison of these old lenses with the lens of a 20-year-old donor (59-20 and $72-20)$ showed obvious peaks at 757 (Tr), $879(\mathrm{Tr}), 1002(\mathrm{Ph}), 1541(\mathrm{Tr})$ and $1602 \mathrm{~cm}^{-1}$ (Ph). In addition, a broad band between 1160 and $1450 \mathrm{~cm}^{-1}$ was observed. This band had its maximum around $1318 \mathrm{~cm}^{-1}$, the vibration wave number of $\mathrm{CH}$. Since these spectra were obtained by subtraction of young from old lenses it must be assumed that upon ageing, lens proteins change in such a way that the Raman signals at the wave numbers indicated are in excess in the young lens. Changes in the amide I and III regions are not obvious or cannot be interpreted.

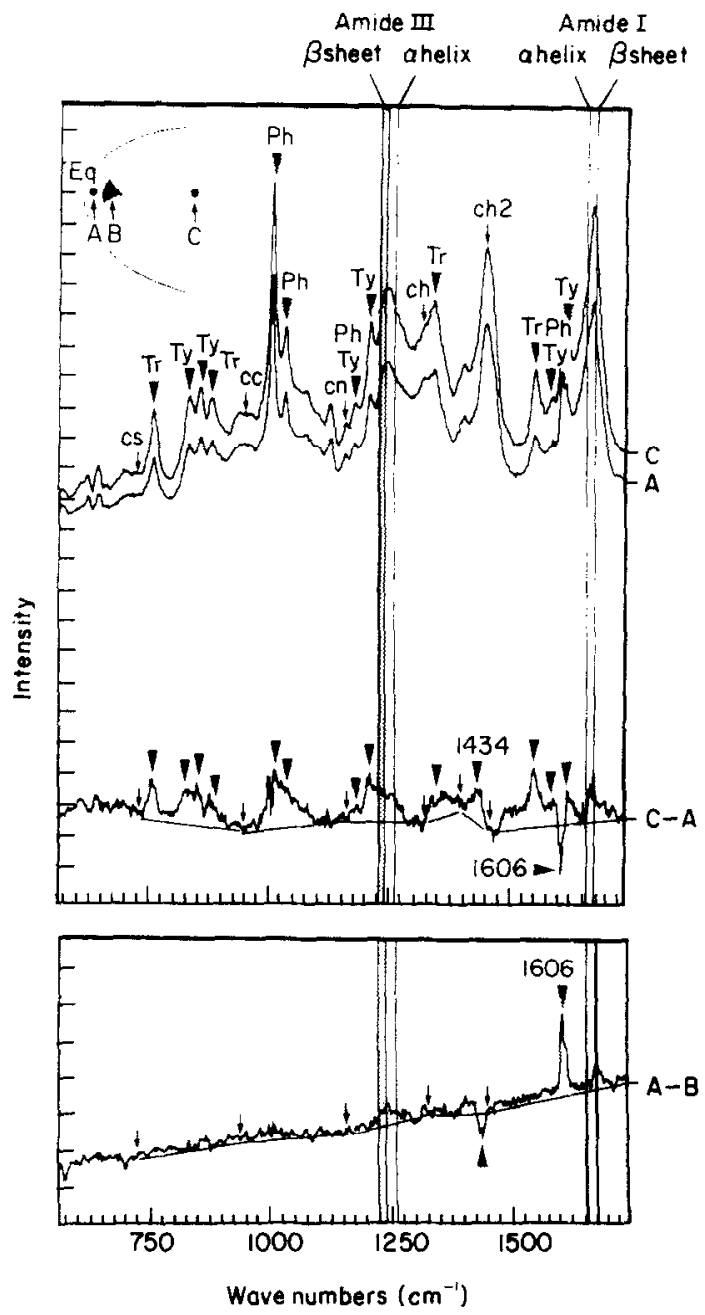

Fig. 5. Panel illustrating the Raman analysis of an opaque spot (B), a closely adjacent transparent site (A) and a nuclear site (C). Although $\mathrm{Ph}$ has a vibration peak at about $1606 \mathrm{~cm}^{-1}$ the shape of the band is such that we are most probably dealing with an artefact.

As indicated in Materials and Methods, lenses with minimal discoloration and no or few opaque spots were selected. Upon stereomicroscopic inspection of the slices, however, two slices showed an opaque spot in the equatorial cortex. Raman analysis of these spots (B) and adjacent transparent regions ( $\mathrm{A}$ and $\mathrm{C}$ ) are summarized in Figs 4 and 5. In the first spot (Fig. 4) no consistent differences between opaque spot and closely adjacent transparent locations were observed for the known protein Raman signals except for a peak at $1002 \mathrm{~cm}^{-1}(\mathrm{Ph})$. This indicates an excess in the spot for this phenylalanine vibration. Comparison of the deep cortical (C) vs. the more superficial cortical locations (A and B) revealed an increased Raman signal in a region between 1425 and $1435 \mathrm{~cm}^{-1}$. The second opaque spot (Fig. 5) was compared with a more superficial (A) and a nuclear (C) location. Except for an excessive Raman signal in the region hetween 1425 and $1435 \mathrm{~cm}^{-1}$ and a deficient signal at $1606 \mathrm{~cm}^{-1}$ in the spot, no differences were observed between the opaque and transparent superficial locations [Figs 5(A)-(B)]. The difference spectrum of 
transparent nuclear and cortical sites $(\mathrm{C}-\mathrm{A})$ in this equatorial region revealed identical changes as observed along the optical axis: excessive Raman signals for the aromatic amino acids and a preponderance of $\beta$-sheet amide signals. Since the absence of clear-cut relevant changes in the opaque spots was puzzling, we wondered whether changes in the orientation of lens proteins could be responsible for the opacification. Therefore, opaque spots and adjacent transparent locations were also analysed using a polarization filter at two perpendicular orientations. In addition, the whole slices were analysed at two perpendicular orientations. However, ilo difference could be observed in this way between opaque and transparent locations.

\section{Discussion}

\section{Methodological Considerations}

Almost all Raman studies devoted to age-related or cataractous changes in the eye lens have used peak ratios of certain specific and relevant assignments to analyse possible alterations in the amino acid composition and/or the secondary and tertiary conformation of the lens proteins (Iriyama et al., 1982/ 1983; Itoh et al., 1983; Ozaki et al., 1983, 1987; Parker, 1983; Yu et al., 1985; De Nagel et al., 1988; Nie et al., 1990; Schyns et al., 1990; Yu, Bando and Kuck, 1990). The analysis of age and cataract-related post-translational changes and of age-related transcriptional/translational changes using this peakratio approach may be biased by local variations in protein content (Ozaki et al., 1983; Otto, de Mul and Greve, 1987). Local variations in absolute protein content have recently been described (Huizinga et al., 1989 ; Siebinga et al., 1991) and the variations proved to be within very short distances. The approach used in this study (i.e. calibration for protein content and subsequent calculation of difference spectra) largely avoids this bias as shown, for example, in Raman studies of DNA-protein interactions (cf. Otto et al., 1987; Otto, 1987: Puppels et al., 1990). The $\mathrm{CH}_{2} / \mathrm{CH}_{3}$ vibration at $1450 \mathrm{~cm}^{-1}$, used in this study as the protein calibration peak, has been rationalized by Fasman et al. (1978) and is generally accepted (Tu, 1982; Ozaki et al., 1983). The advantages of the difference-spectrum approach are that an unbiased picture can be obtained of differences over the whole range of Raman signals and that it is more sensitive to small changes. The present study gives empirical support for the validity and usefulness of the $1450 \mathrm{~cm}^{-1}$ vibration as an internal protein calibration pcak also in eye lenses. In line with biochemical observations, that the major changes in lens proteins occur in early life (Harding, 1981; Hoenders and Bloemendal, 1981; Spector 1985), no relevant differences in nuclear Raman vibrations between 59-year-old and 72-year-old lenses were observed
(Fig. 3). In addition, the fact that between the old lenses and a young lens, one specific backbone or non-aromatic side-chain signal (CH-vibration at $1318 \mathrm{~cm}^{-1}$, Fig. 3) has significantly changed, indicates that the backbone and non-aromatic side-chain vibrations are independent variables and that the absence of this kind of change in the other measurements is real and not due to a trivial subtraction phenomenon. An obvious drawback of age-related studies of human lenses is the inevitable undetermined homogeneity of the tissue due to biological variation and medical history of the donors. Due to legislation the donors are anonymous and therefore the medical history is unknown to us, except for the cause of death. In an epidemiological survey on lenses collected from the Cornea Bank, Amsterdam, no significant correlation between cause of death and lens status was found; not even when the cause of death could be assumed to have been accompanied by long-term drug treatment, e.g. in cases of malignancy (Vrensen and Willekens, 1989). In addition, the lenses selected for the present study were clear with minimal nuclear discoloration and very few incipient opacities and were all of Caucasian origin. Finally, the consistency of the Raman observations make it unlikely that medical history or long-term drug treatment had a significant effect on the proteins.

\section{Differences in Aromatic Amino Acids}

A consistent finding of the present study is the excess of Raman signals for the aromatic amino acids in the proteins of the lens nucleus as compared to those of the superficial cortex. These increased Raman signals may reflect an increased content or a shift in the microenvironment of these amino acids (Tu, 1982; Itoh et al., 1983; Ozaki et al., 1983, 1987; Nie et al. 1990; Schyns et al., 1990; Yu et al., 1990). Since the increase comprises all assigned wave numbers for these aromatic amino acids it seems justifiable to conclude that there is an increased content in aromatic amino acids in the nucleus. This either reflects a post-translational change or a difference in content of the distinct crystallins. Using the Raman vibrations at $574 \mathrm{~cm}^{-1}$ and $760 \mathrm{~cm}^{-1}$ (tryptophan) and at $644 \mathrm{~cm}^{-1}$ (tyrosine), $\mathrm{Yu}$ and East (1975) described an abundance of tryptophan and tyrosine in isolated bovine $\beta$ - and $\gamma$-crystallins compared to $\alpha$-crystallins.

There is substantial biochemical evidence that the nucleus of mammalian and human lenses has an increased $\beta$ - and $\gamma$-crystallin content (Hoenders and Bloemendal, 1981, 1983; Spector, 1985). In addition, amino acid composition analysis revealed that $\beta$ - and $\gamma$-crystallins are rich in tyrosine and tryptophan (Bjork, 1964; Croft, 1972; Coghlan and Augusteyn, 1977; Zigler and Sidbury, 1977; Hoenders and Bloemendal, 1981). Moreover, McAvoy (1981) has reviewed the evidence that the preponderance of $\gamma$ - 
crystallins in the nucleus is due to the expression of this protein at the time of terminal differentiation of lens fibres. This biochemical evidence is in line with the present observation of an increased tryptophan and tyrosine content in the nucleus of the human lens as deduced from the increased Raman signals for these amino acids. Using the weak phenylalanine vibrations at $622 \mathrm{~cm}^{-1}, \mathrm{Yu}$ and East (1975) concluded that phenylalanine is more abundant in $\alpha$-crystallin than in the $\beta$ - and $\gamma$-crystallins of the bovine lens. The present observations of increased Raman signals for the strong 'breathing' vibrations of phenylalanine at 1003 and $1033 \mathrm{~cm}^{-1}$ apparently contradicts the observations made by $\mathrm{Yu}$ and East (1975). This may be due either to a species difference or to the limited use of the I 624/I 644 intensity ratio as suggested by $\mathrm{Tu}$ (1982). In conclusion, the increased Raman responses of tyrosine and tryptophan certainly reflect differences in the side-chain conformation of the nuclear proteins vs. the superficial cortical proteins in the human lens. Most probably these changes are the result of alterations in protein composition, although post-translational changes due to photo-oxidation, as observed by circular dichroism (Andley and Chapman, 1986), cannot be excluded.

As outlined by $\mathrm{Tu}$ (1982), Kitagawa, Azuma and Hamaguchi (1979) and Siamwiza et al. (1975), the Raman signals for the tyrosine doublet at 830 and $855 \mathrm{~cm}^{-1}$ and for the tryptophan doublet at 880 and $760 \mathrm{~cm}^{-1}$ are related to the microenvironment of the tyrosine and tryptophan side chains. The difference spectra for individual lenses give excessive Raman signals for both wave numbers of the doublets indicating that no relevant shift from 'exposed' to 'buried' position of tyrosine and tryptophan residues has occurred. This observation is in accordance with previous studies of tyrosine in non-cataractous mammalian lenses (Itoh et al., 1983; Ozaki et al., 1983, 1987; Nie et al., 1990; Schyns et al., 1990). For tryptophan the literature is more controversial. A decrease in the I 880/I 760 ratio has been observed with age in the ICR-mouse (Ozaki et al., 1987) and in the human lens (Nie et al., 1990). However, no change in this ratio was observed by Itoh et al. (1983) in ageing ICR mice and by Schyns et al. (1990) in cortical vs. nuclear proteins of rabbit lenses.

Since the increase in aromatic amino acids in the nucleus is significant and is additionally a consistent finding, it seems attractive to estimate these changes in quantitative terms and to compare them with biochemically estimated changes in the content of these amino acids. Although in solutions of the aromatic amino acids, the Raman signals will be linearly related to the concentration, the stoichiometry when incorporated in proteins is much more complex and affected, for example by Raman hypo- and hyperchromism. Further studies are in progress which will allow more pertinent conclusions on these quantitative aspects.

\section{Differences in Secondary Conformation}

The conformation of the peptide backbone or secondary structure of proteins has been subject of numerous Raman studies (cf. Tu, 1982). The Amide I and III bands are used to analyse the $\alpha$-helix, random coil, $\beta$-turn or $\beta$-sheet conformation of the proteins. The 'strong' peak at $1673 \mathrm{~cm}^{-1}$ and the band at $1227-1240 \mathrm{~cm}^{-1}$, also observed in the present study. are taken as evidence that lens proteins are largely in the antiparallel $\beta$-pleated sheet conformation (Parker. 1983). This is in accordance with conclusions derived from studies using circular dichroism, optical rotatory dispersion and X-ray crystallography showing that lens proteins mainly are $\beta$-pleated sheets ( $\mathrm{Li}$ and Spector, 1967; Armand, Balazs and Testa, 1970; Li and Spector, 1974; Horowitz, Kabasawa and Kinoshita, 1977; Lindley et al., 1985: Andley and Chapman, 1986; Stiuso, Ragone and Colonna, 1990). These studies also showed, however, that there are differences between the crystallins with no or very minor $\alpha$ helix conformation for $\alpha$-crystallins to about $20 \%$ for $\gamma$-crystallins. The present difference spectra, however. show excessive Raman signals in the nucleus for both $\beta$-sheet assignments at $1673 \mathrm{~cm}^{-1}$ and $1227-$ $1240 \mathrm{~cm}^{-1}$ indicating a more pronounced $\beta$-sheet conformation in the nucleus. As outlined above the nucleus contains excessive $\beta$ - and $\gamma$-crystallins and these proteins proved to have a less pronounced $\beta$ sheet conformation. This means that the shift to $\beta$ conformation reflects a true conformational change upon ageing and is not likely to be due to differences in crystallin content between nucleus and cortex. Since circular dichroism. optical rotatory dispersion and X-ray crystallography studies are carried out on isolated proteins obtained from whole lenses it is not surprising that they did not reveal secondary conformational changes. The discrepancy between the present results and previous studies, using a peak-ratio approach, may have a rational explanation. In proteins with a major $\beta$-sheet conformation the $\alpha$ helix and random coil signals at 1660 and $1645 \mathrm{~cm}^{-1}$ for Amide I and at $>1264$ and $1248 \mathrm{~cm}^{-1}$ for Amide III (Parker, 1983) will be 'weak' and present as shoulders on the $\beta$-sheet peaks. Therefore, the peakratios of these assignments will not be particularly sensitive and discriminative.

\section{Conformational Changes Among Lenses of Varying Age}

On account of technical considerations, i.e. exactly identical instrumental conditions, the comparisons have been restricted to three lenses of different ages. Despite the fact that these are preliminary observations. some conclusions can be drawn since the results are consistent. In Fig. 3 it is shown that the spectral differences among nuclear proteins are much morc pronounced than among cortical proteins. This seems in good agreement with the common fact that 
new proteins are synthesized throughout life and that thus the actual age of superficial proteins will be about the same irrespective of the age of the lens. It also indicates that no gross age-related transcriptional/ translational changes regarding the secondary and tertiary conformation of proteins occur. The differences between young and old nuclear proteins are puzzling. The neutral difference spectrum between the 59- and 72-year-old lenses is not surprising as no gross alterations are expected between clear lenses of these ages. The lower tryptophan, tyrosine and phenylalanine content of old nuclear proteins may reflect a change in the crystallin composition of old vs. young lenses. The excessive Raman signals in the young lens in the $1160-1450 \mathrm{~cm}^{-1}$ region, with a maximum for the $\mathrm{CH}$-vibrations at $1318 \mathrm{~cm}^{-1}$, is most enigmatic. This region includes known assignments for tyrosine/phenylalanine $\left(1177 \mathrm{~cm}^{-1}\right)$, tyrosine $\left(1209 \mathrm{~cm}^{-1}\right)$. Amide III $\left(1240-1270 \mathrm{~cm}^{-1}\right)$. tryptophan $\left(1342 \mathrm{~cm}^{-1}\right)$ and $\mathrm{CO}_{2}\left(1404 \mathrm{~cm}^{-1}\right)$. As the other phenylalanine and tryptophan peaks also show significant changes it might be that this band also reflects the higher content of these aromatic amino acids in the young lens nucleus. The observations of a lower tryptophan content in the nucleus of old lenses are to some extent in agreement with a recent study by $\mathrm{Nie}$ et al. (1990). With respect to changes in the secondary conformation, no definite conclusions can be drawn since the Amide III peaks are masked by the deep $1160-1450 \mathrm{~cm}^{-1}$ band. The absence of a clear-cut peak at $1673 \mathrm{~cm}^{-1}$ (Amide I) makes it rather unlikely, however, that there are gross differences between young and old nuclear proteins regarding this secondary conformation. This is also in agreement with the observations made by Nie et al. (1990).

\section{Protein Conformation Opaque Regions}

Previous epidemiological and ultrastructural studies have shown that a majority of optically non-opaque old ( $>40$ years) lenses have small opacities in their equatorial cortex in the form of radial or circular shades (Willekens. Kappelhof and Vrensen, 1987; Vrensen, Kappelhof and Willekens, 1990; Vrensen and Willekens, 1990). These studies proved that these small early opacities are filled with protein-containing vacuoles. The opacities analysed in the present study are representative examples of the ultrastructurally well-defined and uniform equatorial radial (Fig. 4) and circular shades (Fig. 5). Critical, with respect to the comparison of the npaque and clear regions, is the attenuation of laser intensity due to light scatter and accordance in the opacities. This leads to a lower Raman signal per unit of time but not to an increase of noise since access of elastically scattered light to the detector is prevented. The attenuation of intensity is compensated for by an increased measuring time. A major conclusion from the Raman difference spectra is that there are no gross differences in conformation between these opacities and closely adjacent clear zones. The difference spectrum between nuclear and cortical sites [Figs 5(C)-(A)] reveals increased Raman signals for all aromatic amino acid wave numbers indicating an increased content of these amino acids in the nucleus. This is in accordance with the observations along the optical axis. One puzzling observation in both opacities is an excessive Raman signal in the deeper cortex and nucleus in the $1425-1435 \mathrm{~cm}^{-1}$ region. Several bands contribute to this region: the $\delta(\mathrm{N}-\mathrm{H})$ band of tryptophan (Yu and East, 1975) and the carboxyl group vibration of acidic amino acids (Dollish, Fateley and Bentley, 1974). The decrease of this peak in the superficial equatorial cortex can be rationalized for both options. Posttranslational modifications have been described for tryptophan and asparagine. Photo-oxidation of tryptophan to $\delta$ - $\mathrm{N}^{\prime}$-formylkynurenine has been verified leading to a change in the five-numbered ring and therefore also to a change in the $\delta-\mathrm{N}-\mathrm{H}$ vibration (Dillon, 1985; Zigman, 1985). Deamidation of asparagine to aspartic acid is a well-known ageing phenomenon for lens proteins (Harding, 1981; Hoenders and Bloemendal, 1981). Both changes are related to the actual age of the protein and the tryptophan change also depends on radiation with light. Since equatorial proteins are young and still largely protected from light irradiation it is not surprising that the difference in this region, whether due to photo-oxidation of tryptophan or to deamidation of asparagine, is most pronounced in the equatorial region. Upon closer examination of the difference spectra between nucleus and cortex along the optical axis a difference peak at $1425 \mathrm{~cm}^{-1}$ is also observed in most of the lenses investigated (Figs 1 and 2).

\section{Conclusions and Outlook}

The Raman analysis of human lenses of varying age, using the difference spectrum approach, reveals differences in the conformation of the crystallins between nucleus and cortex, i.e. an increased content of aromatic amino acid side chains and a more pronounced $\beta$-sheet conformation in the nucleus. It is most likely that these differences reflect a change in the crystallin composition and a true age-related posttranslational alteration, respectively. The study also revealed a difference between old and young nuclear proteins especially with respect to a higher content of aromatic amino acids in the young nucleus. Small early opacities do not exhibit gross changes in protein conformation in comparison with adjacent clear zones. The cortical vs. nuclear spectra support the biochemical observations of tryptophan photo-oxidation to $\delta$ - $\mathrm{N}^{\prime}$-formylkynurenine and/or deamidation of asparagine to aspartic acid. The present study shows that Raman analysis, especially when using the difference-spectrum approach, is a powerful, highly discriminative and sensitive method for the study of 
protein conformational changes. Since, in addition. the microspectroscopic set-up has a pronounced local resolution, this kind of analysis will be a useful tool for future investigations of protein alterations in advancing cataractous changes and of precise local post-translational and transcriptional/translational changes of human lens proteins.

\section{Acknowledgements}

The authors are indebted to Dr E. Pels and her co-workers of the Cornea bank, Amsterdam, for the human lenses; to $\mathrm{Mr}$ B. Willekens for technical assistance; and to Mrs Y. Heijens and Mrs A. de Wolf for secretarial assistance.

\section{References}

Andley, U. P. and Chapman, S. F. (1986). Conformational changes of bovine lens crystallins in a photodynamic system. Photochem. Photobiol. 44, 67-74.

Armand, G., Balazs, E. A. and Testa, M. (1970). Separation and partial characterization of two proteins from fraction B of calf lens. Exp. Eye Res. 10, 143-50.

Barron, B. C., Yu, N.-T. and Kuck, J. F. R. (1987). Tryptophan Raman/457.9-nm-excited fluorescence of intact guinea-pig lenses in aging and ultraviolet light. Invest. Ophthalmol. Vis. Sci. 28, 815-21.

Björk. I. (1964). Studies on $\gamma$-crystallin from calf lens. Exp. Eye Res. 3, 254-61.

Bot, A. C. C., Huizinga, A., de Mul, F. F. M., Vrensen, G. F. J. M. and Greve, J. (1989). Raman microspectroscopy of fixed rabbit and human lenses and lens slices: new potentialities. Exp. Eye Res. 49. 161-9.

Coghlan, S. D. and Augusteyn, R. C. (1977). Changes in the distribution of proteins in the aging human lens. Exp. Eye Res. 25, 603-11.

Croft, L. R. (1972). The amino acid sequence of $\gamma$-crystallin (fraction II) from calf lens. Biochem. J. 128, 961-70.

DeNagel, D. E., Bando, M., Yu, N.-T. and Kuck, J. F. R. (1988). A Raman study of disulfide and sulfhydryl in the Emory Mouse cataract. Invest. Ophthalmol. Vis. Sci. $29,823-6$.

Dillon, J. (1985). Photochemical mechanisms in the lens. In The Ocular Lens. (Ed. Maisel, H.). Pp. 349-66. Marcel Dekker: New York.

Dollish, F. R., Fateley, W. G. and Bentley, F. F. (1974). Characteristic Raman Frequencies of Organic Compounds. John Wiley and Sons: New York.

Fasman, G. D., Itoh, K.. Liu, C. S. and Lord, R. (1978). Laserexcited Raman spectroscopy of biomolecules. XI. Conformational study of poly (L-valine) and copolymers of L-valine and L-alanine. Biopolymers 17, 125-43.

Harding. J. (1981). Changes in lens proteins in cataract. In Molecular and Cellular Biology of the Eye Lens. (Ed. Bloemendal, H.). Pp. 327-65. John Wiley and Sons: New York.

Hoenders, H. J. and Bloemendal, H. (1981). Aging of lens proteins. In Molecular and Cellular Biology of the Eye Lens. (Ed. Bloemendal, H.). Pp. 279-326. John Wiley and Sons: New York.

Hoenders, J. and Bloemendal, H. (1983). Aging of the eye lens. J. Gerontol. 38, 278-86.

Horwitz. J., Kabasawa, I. and Kinoshita, J. H. (1977). Conformation of Gamma-crystallins of the calf lens: effects of temperature and denaturing agents. Exp. Eye Res. 25, 199-208.
Huizinga, A., Bot, A. C. C., de Mul, F. F. M.. Vrensen, (i. F. J. M. and Greve, J. (1989). Local variation in absolute water content of human and rabbit eye lenses measured by Raman microspectroscopy. Exp. Eye Res. 48. $487-96$.

Iriyama, K.. Mizuno, A., Ozaki, Y., Itoh, K. and Matsuzaki, H. (1982/1983). An application of laser Raman spectroscopy to the study of a hereditary cataractous lens; on the Raman band for a diagnostic marker of cataractous signatures. Curr. Eye Res. 2, 489-92.

Itoh, K., Ozaki, Y., Mizuno, A. and Iriyama, K. (1983). Structural changes in the lens proteins and hereditary cataracts monitored by Raman spectroscopy. Biochemistry 22, 1773-83.

Kitagawa, T., Azuma, T. and Hamaguchi, K. (1979). The Raman spectra of Bence-Jones proteins: disulfide stretching frequencies and dependence of Raman intensity of tryptophan residues on their environment. Biopolymers 18, 451-65.

Li. L.-K. and Spector, A. (1967). The optical rotatory dispersion and circular dichroism of calf lens $\alpha$ crystallin. J. Biol. Chem. 242, 3234-6.

Li, L.-K. and Spector, A. (1974). Circular dichroism and optical rotatory dispersion and the aggregates of purified polypeptides of alpha-crystallin. Exp. Eye Res. 19. 49-57.

Lindley, P. F., Narebor, M. E., Summers, L. J. and Wistow, G. J. (1985). The structure of lens proteins. In The Ocular Lens. (Ed. Maisel, H.). Pp 123-67. Marcel Dekker: New York.

McAvoy, J. W. (1981). Developmental biology of the lens. In Mechanisms of Cataract Formation in the Human Lens. (Ed. Duncan, G.). Pp. 7-46. Academic Press: London.

Nie, S., Bergbauer, K. L., Kuck, J. F. R. Jr. and Yu, N.-T. (1990). Near-infrared Fourier transform Raman spectroscopy in human lens research. Exp. Eye Res. 51, 619-23.

Otto, C. (1987). Raman and surface enhanced Raman spectroscopy of $\alpha$-helix destabilizing proteins and nucleotides. Ph.D. Thesis, Technical University of Twente. The Netherlands.

Otto. C.. de Mul. F. F. M. and Greve, J. (1987). A Raman spectroscopic study of the interaction between nucleotides and the DNA binding protein GP 32 of bacteriophage T'4. Biopolymers 26, 1667-89.

Ozaki. Y., Mizuno, A., Itoh, K.. Matsushima, S. \& Iriyama, K. (1987). Raman spectroscopic study of cataract formation: Emory Mouse cataract. Appl. Spectrosc. 41. $597-604$.

Ozaki. Y.. Mizuno, A., Yoshiura, M., Iwamoto, T. and Iriyama, K. (1983). Raman spectroscopic study of agerelated structural changes in the lens proteins of an Intact Mouse lens. Biochemistry 22, 6257-9.

Parker. F. S. (1983). Application of Infrared, Raman, and Resonance Raman Spectroscopy in Biochemistry. Ch. 4. Plenum Press: New York.

Puppels, G. J.. Colier. W., Olminkof, J. H. F., Otto, C., de Mul, F. F. M. and Greve, J. (1991). Description and performance of a highly sensitive confocal Raman microspectrometer. J. Raman Spectrosc. 22, 217-25.

Puppels, G. J., de Mul, F. F. M., Otto, C., Greve, J., RobertNicoud, M., Arndt-Jovin, D. J. and Jovin, T. M. (1990). Studying single living cells and chromosomes by confocal Raman Inicrospectroscopy. Nature 347. 301-3.

Schachar, R. A. and Solin. S. A. (1975). The microscopic protein structure of the lens with a theory for cataract formation as determined by Raman spectroscopy of intact bovine lenses. Invest. Ophthalmol. Vis. Sci. 14. 380-96. 
Schyns, M. W. R. J., Huizinga, A., Vrensen, G. F. J. M., de Mul, F. F. M. and Greve, J. (1990). Paraformaldehyde fixation and some characteristics of lens proteins as measured by Raman microspectroscopy. Exp. Eye Res. 50, 331-3.

Siamwiza, M. N., Lord, R. C., Chen, M. C., Tahamatsu, T., Harada, I., Matsuura, H. and Shimanouchi, T. (1975). Interpretation of the doublet at 850 and $830 \mathrm{~cm}^{-1}$ in the Raman spectra of tyrosyl residues in proteins and certain model compounds. Biochemistry 14, 4870-6.

Siebinga, I., Vrensen. G. F. J. M.. de Mul, F. F. M. and Greve. J. (1991). Age-related changes in local water and protein content of human eye lenses measured by Raman microspectroscopy. Exp. Eye Res. 53, 233-9.

Spector, A. (1985). Aspects of the biochemistry of cataract. In The Ocular Lens. (Ed. Maisel, H.). Pp. 405-38. Marcel Dekker: New York.

Stiuso, P., Ragone, R. and Colonna. G. (1990). Molecular organization and structural stability of $\beta$-crystallin from cal lens. Biochemistry 29, 3929-36.

Tu, A. T. (1982). Raman Spectroscopy in Biology; Principles and Applications. Ch. 3. John Wiley and Sons: New York.

Vrensen, G., Kappelhof, J. and Willekens, B. (1990). Morphology of the aging human lens. II. Ultrastructure of clear lenses. Lens Eye Tox. Res. 7, 1-30.

Vrensen, G. and Willekens, B. (1989). Classification and prevalence of early senile lens opacities in human donor eycs. In Risk Factors for Cataract Development. Dev. Ophthalmol. Vol, 17. (Eds Hockwin, 0., Sasaki, K. and Leske, M. C.). Pp. 181-7. Karger: Basel.
Vrensen, G. and Willekens, B. (1990). Biomicroscopy and scanning electron microscopy of early opacities in the aging human lens. Invest. Ophthalmol. Vis. Sci. 31, 1582-91.

Willekens, B., Kappelhof, J. and Vrensen, G. (1987). Morphology of the aging human lens. I. Biomicroscopy and biometrics. Lens Res. 4. 207-30.

Yu. N.-T., Bando, M. and Kuck, J. F. R. Jr. (1985). Fluorescence/Raman intensity ratio for monitoring the pathologic state of human lens. Invest. Ophthalmol. Vis. Sci. 26, 97-101.

Yu, N.-T., Bando, M. and Kuck, J. F. R. Jr. (1990). Localization of UV-induced changes in mouse lens. Exp. Eye Res. 50, 327-9.

Yu, N.-T.. DeNagel, D. C., Ho, D. J. Y. and Kuck, J. F. R. (1987). Ocular lenses. In Biologicul Applicutions of Rumun Spectroscopy. Vol. 1. (Ed. Spira, T. G.). Pp. 47-80. John Wiley and Sons: New York.

Yu, N.-T., DeNagel, D. C., Pruett, P. L. and Kuck, J. F. R. Jr. (1985). Disulfide bond formation in the eye lens. Proc. Natl. Acad. Sci. U.S.A. 82, 7965-8.

Yu, N.-T. and East, E. J. (1975). Laser Raman spectroscopic studies of ocular lens and its isolated protein fractions. J. Biol. Chem. 250, 2196-202.

Zigler, J. S. and Sidbury, J. B. Jr. (1977). Studies on lens proteins from the smooth dogfish. Ophthalmic Res. 8. $92-8$.

Zigman. S. (1985). Photobiology of the lens. In The Ocular Lens. (Ed. Maisel, H.). Pp. 301-47. Marcel Dekker: New York. 